\title{
Cangxitongbi capsules protect the articular cartilage in the rat knee through the long non-coding RNA HOTAIR/p38MAPK pathway
}

\author{
Xu-Yu Song ${ }^{1 \#}$, Min Zhao ${ }^{2 \#}$, Peng Zhang ${ }^{1 \#}$, Ling-Sen Yang ${ }^{3}$, Rong-Xiu Bi ${ }^{3}$, Wen-Peng Xie ${ }^{3}$ \\ ${ }^{1}$ First Clinical College, Shandong University of Traditional Chinese Medicine, Jinan, China; ${ }^{2}$ Department of Paediatrics, Shandong Maternal and \\ Child Health Hospital, Jinan, China; ${ }^{3}$ Department of Orthopedics, Affiliated Hospital of Shandong University of Traditional Chinese Medicine, \\ Jinan, China \\ Contributions: (I) Conception and design: XY Song, WP Xie, RX Bi; (II) Administrative support: RX Bi; (III) Provision of study materials or patients: \\ XY Song, WP Xie; (IV) Collection and assembly of data: XY Song, P Zhang, M Zhao; (V) Data analysis and interpretation: P Zhang, M Zhao, LS \\ Yang; (VI) Manuscript writing: All authors; (VII) Final approval of manuscript: All authors. \\ \#These authors contributed equally to this work. \\ Correspondence to: Dr. Wen-Peng Xie; Prof. Rong-Xiu Bi. Department of Orthopedics, Affiliated Hospital of Shandong University of Traditional \\ Chinese Medicine, Jinan, China. Email: xiewenpeng0925@163.com; birongxiu@hotmail.com.
}

\begin{abstract}
Background: Knee osteoarthritis (KOA) is a leading cause of chronic pain and disability, and as such, it poses a significant economic burden. Traditional Chinese medicine (TCM), as well as complementary and alternative medicine, can offer safe and effective treatments for KOA. Cangxitongbi (CXTB) capsule is a Chinese patented medicine for KOA treatment and has a remarkable curative effect. This article evaluated the effects and mechanisms of CXTB in protecting joint cartilage in vivo.
\end{abstract}

Methods: The KOA model was constructed in rats using the modified Hulth method. CXTB (35 mg/kg) was administered intragastrically for 4 weeks. Hematoxylin and eosin (HE) staining of the knee articular were performed to evaluate the efficiency of CXTB. Western blot analysis, quantitative polymerase chain reaction (qPCR), and enzyme-linked immunosorbent assay (ELISA) were used to investigate the protective mechanisms of CXTB in joint cartilage.

Results: CXTB effectively improved the morphological structure of the cartilage and bone in the knee joint by enhancing autophagy and regulating the expression of related protease and inflammatory factors. Furthermore, CXTB downregulated the expression of the long non-coding RNA (lncRNA) Hox transcript antisense intergenic RNA (HOTAIR) and inhibited the activation of the p38MAPK pathway. Conversely, overexpression of lncRNA HOTAIR suppressed the protective effects of CXTB on the knee joint.

Conclusions: CXTB capsules can protect the knee articular cartilage in rats through the lncRNA HOTAIR/p38MAPK pathway.

Keywords: Cangxitongbi capsules (CXTB capsules); long non-coding RNA Hox transcript antisense intergenic RNA (lncRNA HOTAIR); articular cartilage; knee osteoarthritis (KOA)

Submitted Nov 22, 2021. Accepted for publication Dec 23, 2021.

doi: 10.21037/atm-21-6539

View this article at: https://dx.doi.org/10.21037/atm-21-6539

\section{Introduction}

Knee osteoarthritis (KOA) is an increasingly prevalent disease characterized by progressive joint destruction, chronic joint pain, deformity, dysfunction, joint apraxia, and even disability (1). The high incidence of KOA exerts a heavy burden on society and the economy. KOA is multifactorial disease that may be affected by joint injury, aging, and trauma (2). Due to the limitations of Western 
medicine for the treatment of KOA (3), traditional Chinese medicine (TCM) and other complementary and alternative medicines have become increasingly utilized.

It is recognized that TCM can regulate the overall health of the whole body and has been shown to be relatively safe (4). Cangxitongbi (CXTB) capsules contain a Chinese herbal compound and has been widely used to treat KOA and KOA-related symptoms with promising efficacy (5).

This current study investigated the efficacy and pharmacology of CXTB by examining its effects on autophagy and cartilage structure in a rat KOA model. We present the following article in accordance with the ARRIVE reporting checklist (available at https://dx.doi. org/10.21037/atm-21-6539).

\section{Methods}

\section{Drug preparation}

CXTB (\#Z20130043) was purchased from the TCM Pharmacy of the Affiliated Hospital of Shandong University of Traditional Chinese Medicine (Jinan, China). The drug was ground into a fine powder and dissolved in saline to reach a concentration of $35 \mathrm{mg} / \mathrm{mL}$.

\section{Adeno-associated virus (AAV) preparation}

The AAV overexpressing the long non-coding RNA (lncRNA) Hox transcript antisense intergenic RNA (HOTAIR) (AAV-HOTAIR) and the non-coding AAV (AAV-control) were purchased from Genomeditech Co., Shanghai, China. All viruses are packed separately and stored at $-80^{\circ} \mathrm{C}$ and thawed before use.

\section{Animals}

A protocol was prepared before the study without registration. Animal experiments were approved by the Animal Ethics Committee of Affiliated Hospital of Shandong University of Traditional Chinese Medicine (AWE-2019-043), in compliance with Guide for the Care and Use of Laboratory Animals, 8th edition, for the care and use of animals.

A total of 50 4-week-old male specific pathogen free (SPF) rats, weighing 221 18 g, were purchased from Jinan Pengyue Experimental Animal Breeding Co., Ltd. [certificate: SCXK(LU)20190003]. Animals were housed in the Center for Animal Experimentation with a 12-hour light/dark cycle at a temperature of $25 \pm 1{ }^{\circ} \mathrm{C}$ and humidity of
$50 \% \pm 5 \%$, and allowed access to the diet ad libitum.

\section{Modeling and administration}

The rats were randomly divided into the negative control group $(\mathrm{NC}, \mathrm{n}=10)$ or the KOA group (KOA, $\mathrm{n}=40)$. The KOA rats were anesthetized with $1 \%$ pentobarbital $(50 \mathrm{mg} / \mathrm{kg})$ and treated with a modified Hulth procedure. Briefly, the left knee anterior cruciate and medial collateral ligaments were transected and the medial meniscus was resected. The NC rats were fed normally without treatment.

After 4 weeks, radiographs of the knees were taken to confirm the condition of the damaged joint. Rats in the KOA group that showed unsmooth and sclerotic knee joint surfaces compared to rats in the NC group were considered KOA rats. All 40 rats were screened.

Subsequently, the $40 \mathrm{KOA}$ rats were randomly divided into the model group (intragastrically treated with the same amount of saline as other groups), the CXTB group (intragastrically administered $35 \mathrm{mg}$ crude CXTB drug/ $\mathrm{kg} / \mathrm{d}$, calculated according to the Meeh-Rubner formula and direct conversion algorithm), the CXTB + AAV-HOTAIR group (intragastrically administered $35 \mathrm{mg}$ crude CXTB $\mathrm{drug} / \mathrm{kg} / \mathrm{d}$ and injected with $1 \times 10^{10} \mathrm{vg}$ AAV-HOTAIR into the left knee joint cavity once), and the CXTB + AAV-control group (intragastrically administered $35 \mathrm{mg}$ crude CXTB $\mathrm{drug} / \mathrm{kg} / \mathrm{d}$ and injected with $1 \times 10^{10} \mathrm{vg} \mathrm{AAV}$-control into the left knee joint cavity once). There were 10 rats in each group. Rats in the NC group were treated as for the model group. After 4 weeks of treatment, all rats were euthanatized with $1 \%$ pentobarbital $(100 \mathrm{mg} / \mathrm{kg})$ and the articular cartilages of the left knee were removed. Peripheral blood was collected via venipuncture and centrifuged to obtain the plasma for subsequent experiments.

\section{Hematoxylin and eosin (HE) staining}

Cartilage tissues were fixed with $4 \%$ paraformaldehyde, decalcified with $10 \%$ ethylene diamine tetraacetic acid$2 \mathrm{Na}$ (EDTA-2Na), and embedded in paraffin. Sections with a thickness of $5 \mu \mathrm{m}$ were prepared and stained with HE (Solarbio, Beijing, China). Slides were observed microscopically and evaluated blinded.

\section{Transmission electron microscopy (TEM)}

The cartilage tissues were rapidly cut into $1-2 \mathrm{~mm}^{3}$ pieces 
and immersed in a fixative solution (Servicebio, G1102, Wuhan, China) for $2-4$ hours at $4{ }^{\circ} \mathrm{C}$. The autophagosomes were visualized by electron microscopy.

\section{Western blot}

The cartilage tissues were ground and lysed in lysis buffer (Beyotime Biotechnology Company, Nanjing, China). The protein concentration was determined using the BCA protein assay kit (Beyotime Biotechnology Company, Nanjing, China) and $50 \mu \mathrm{g}$ protein samples were separated via sodium dodecyl sulfate polyacrylamide gel electrophoresis (SDS-PAGE) and transferred to polyvinylidene fluoride (PVDF) membranes. Membranes were blocked in $5 \%$ non-fat dry milk in Tris buffered saline containing Tween-20 (TBST), followed by incubation with the primary antibodies (rabbit anti-MMP 13 antibody, Servicebio, GB11247-1, 1:1,000, Wuhan, China; rabbit anti-TIMP 1 antibody, Abcam, ab109125, 1:1,000, Cambridge, UK; rabbit anti-LC3 antibody, Servicebio, GB11124, 1:1,000, Wuhan, China; rabbit anti-p38 antibody, Abcam, ab32142, 1:1,000, Cambridge, UK; rabbit antip-p38 antibody, Wanleibio, WL03428, 1:1,000, Shenyang, China; mouse anti- $\beta$-actin antibody, Servicebio, GB12001, 1:1,000, Wuhan, China). After washing with TBST, the membranes were incubated with a horseradish peroxidaseconjugated secondary antibody (goat anti-rabbit IgG, Servicebio, GB23303, 1:5,000, Wuhan, China; goat antimouse IgG, Servicebio, GB23301, 1:5,000, Wuhan, China) for 1 hour at room temperature. The blots were visualized with enhanced chemiluminescence solution (ECL) and the Western blot detection system (Image Quant, LAS500, GE Company, Marlborough, MA, USA). The density values of the bands were quantified using the Image $\mathrm{J}$ software (National Institutes of Health, Bethesda, MD, USA). $\beta$-actin was used as an internal control.

\section{Quantitative polymerase chain reaction (qPCR)}

Total RNA was extracted with TRIzol reagent (Invitrogen Corp., Carlsbad, CA, USA), quantified with a NanoDrop 2000c spectrophotometer (Thermo Fisher Scientific, Waltham, MA, USA), and reverse-transcribed using the Sparkjade Reverse Transcriptase Kit (Sparkjade, Qingdao, China). The expression of the relevant mRNAs was determined using the $2^{-\Delta \Delta C t}$ method. GAPDH was used as an internal control. The primers used are listed as follows: MMP13: (F: 5'-TGCATACGAGCATCCATCCC-3' and
R: 5'-CGTGTCCTCAAAGTGAACCGC-3'); TIMP1: (F: 5'-GCCTCTGGCATCCTCTTGTTG-3' and R: 5'-GAACCGGAAACCTGTGGCAT-3'); IncRNA HOTAIR: (F: 5'-GGTCCTTCTCCACTTTGCTGC-3' and R: 5'-GCTGGTTTGGGTTGTAGCACTT-3'); p38: (F: 5'-ACCACGACCCTGATGATGAGC-3' and R: 5'-TAGGTCAGGCTCTTCCATTCGT-3'); and GAPDH (F: 5'-CTGGAGAAACCTGCCAAGTATG-3' and R: 5'-GGTGGAAGAATGGGAGTTGCT-3').

\section{Enzyme-linked immunosorbent assay (ELISA)}

Peripheral blood samples collected via venipuncture were centrifuged to obtain the plasma. The expression of interleukin (IL)-1 $\beta$ (Multi sciences, EK301B/3-01, Hangzhou, China) and tumor necrosis factor (TNF)- $\alpha$ (Invitrogen, 88-7340, CA, USA) were assessed by ELISA according to the manufacturer's instructions.

\section{Statistical analysis}

All data are shown as mean \pm standard deviation $(\bar{x} \pm \mathrm{S})$ and were calculated using one-way analysis of variance (ANOVA) using the SPSS version 22.0 software (IBM Corp., Armonk, NY, USA). A P value $<0.05$ was considered statistically significant.

\section{Results}

\section{KOA was successfully induced in rats}

Radiographs of the knees of rats in the KOA group showed multiple hyperosteogenic, sclerotic, and cystic changes. Furthermore, the surface of the knee joint in KOA rats was rough and unsmooth while the articular surface of the rats in the NC group was smooth with normal joint space and no bony destruction. These observations suggested that the KOA model was successfully constructed in these rats (Figure 1).

\section{CXTB attenuates cartilage injury by downregulating the IncRNA HOTAIR}

The expression of the IncRNA HOTAIR was detected using qPCR (Figure 2A). Rats in the KOA model group showed upregulated expression of HOTAIR in the articular cartilage tissue compared to rats in the $\mathrm{NC}$ group $(\mathrm{P}<0.05)$. In the CXTB group, the expression of HOTAIR was 
Page 4 of 10

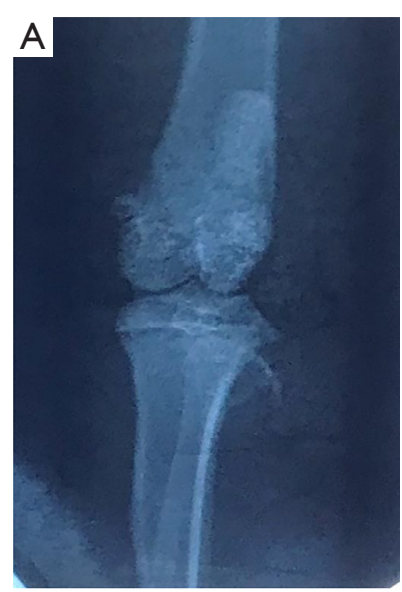

$\mathrm{NC}$

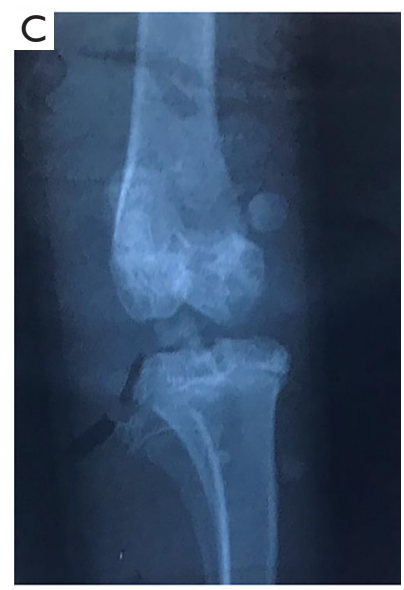

Model

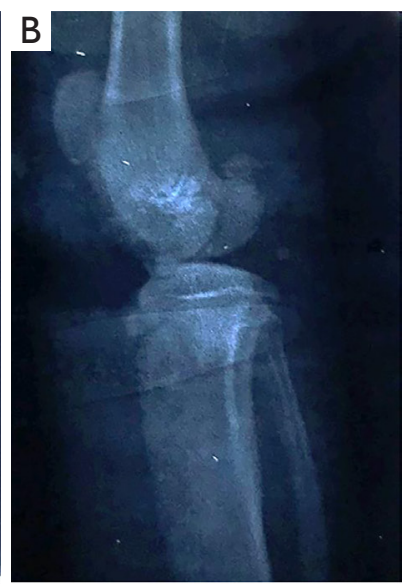

$\mathrm{NC}$

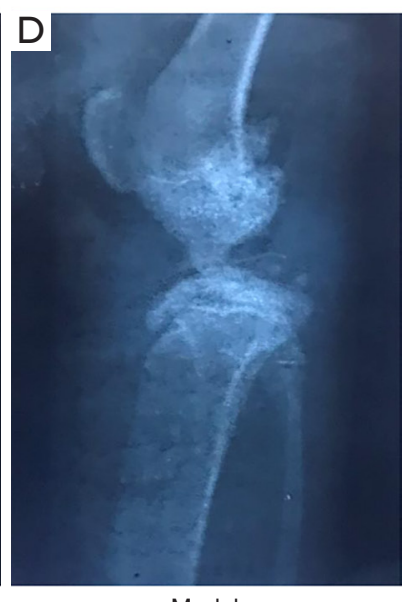

Model
Figure 1 Radiographs of the knee joint. (A,B) Radiographs of the knee joint from rats in the NC group. (C,D) Radiographs knee joints from rat in the model group. NC, negative control.

obviously downregulated compared with the model group $(\mathrm{P}<0.05)$ and the CXTB + AAV-HOTAIR group $(\mathrm{P}<0.05)$. There was no significant difference in HOTAIR expression between the CXTB group and the CXTB + AAV-control group $(\mathrm{P}>0.05)$.

In the model rats, HE staining demonstrated cracks and blood vessels passing through cartilage tissue, the cartilage layers were abnormal, and the tide line was lost. However, administration of CXTB improved the morphology of the lesional cartilage. In the CXTB + AAV-HOTAIR group, the degree of cartilage damage was more aggravated compared with the CXTB group, the cartilage surface was rough with more obvious cracks and blood vessels passing through cartilage tissue (Figure $2 B-2 F$ ).

The Osteoarthritis Research Society International

\section{Song et al. CXTB regulates IncRNA HOTAIR/p38MAPK in vivo}

(OARSI) score was used to judge the severity of cartilage damage (Figure 2G). The OARSI score of the model group was higher than that of the $\mathrm{NC}$ group $(\mathrm{P}<0.05)$, suggesting that the cartilage was severely damaged and the modeling was successful. Meanwhile, the score of the CXTB group was significantly lower than that of the model group $(\mathrm{P}<0.05)$ and the CXTB + AAV-HOTAIR group $(\mathrm{P}<0.05)$. However, there was no difference in the scores between the CXTB group and the CXTB + AAV-control group $(\mathrm{P}>0.05)$.

The protein and mRNA expression of the cartilage damage-related proteins matrix metallopeptidase 13 (MMP13) and tissue inhibitor matrix metalloproteinase 1 (TIMP1) were detected with Western blot and qPCR (Figure $2 \mathrm{H}-2 \mathrm{~L}$ ). In the model group, the protein expression of MMP13 was the highest among all groups $(\mathrm{P}<0.05)$. The expression of MMP13 in the CXTB group was lower than that of the model group $(\mathrm{P}<0.05)$ and the $\mathrm{CXTB}+\mathrm{AAV}$ HOTAIR group $(\mathrm{P}<0.05)$. Similarly, there was no difference between the CXTB group and the CXTB + AAV-control group $(\mathrm{P}>0.05)$. The expression of TIMP1 showed an inverse trend to that of MMP13 and this was not surprising as TIMP1 is an antagonist protein of MMP13. These results were confirmed by qPCR (Figure $2 K, 2 L$ ).

The above data suggested that CXTB exerted a protective effect on articular cartilage and this effect was achieved through downregulation of HOTAIR expression.

\section{CXTB suppresses the inflammatory response by downregulating lncRNA HOTAIR}

ELISAs were performed to detect the levels of the inflammatory markers IL- $1 \beta$ and TNF- $\alpha$ in the peripheral blood serum (Figure 3). The levels of serum IL-1 $\beta$ and TNF- $\alpha$ were significantly higher in model rats compared to rats in the NC group $(\mathrm{P}<0.05)$. The serum expression of IL-1 $\beta$ and TNF- $\alpha$ was lower in the CXTB group compared to the model group $(\mathrm{P}<0.05)$ and the CXTB + AAV-HOTAIR group $(\mathrm{P}<0.05)$. However, IL-1 $\beta$ and TNF- $\alpha$ expression was similar between the CXTB group and the CXTB + AAVcontrol group $(\mathrm{P}>0.05)$. These results suggested that $\mathrm{CXTB}$ suppressed inflammation by decreasing the expression of IL$1 \beta$ and TNF- $\alpha$, which was achieved by downregulating the expression of lncRNA HOTAIR.

\section{CXTB enbances autophagy by downregulating lncRNA HOTAIR}

TEM of the cartilage tissue revealed that the articular 

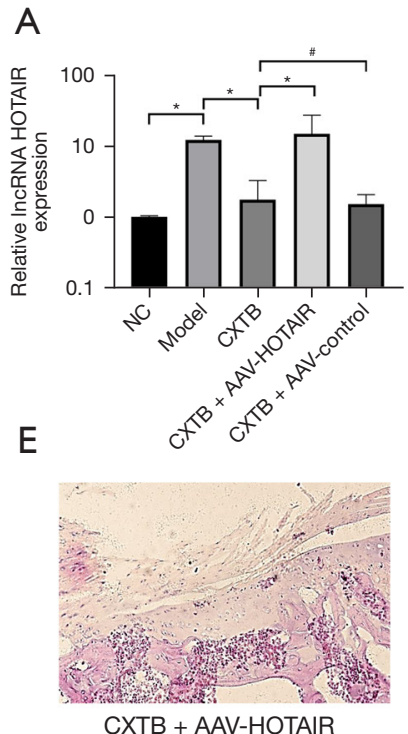

CXTB + AAV-HOTAIR

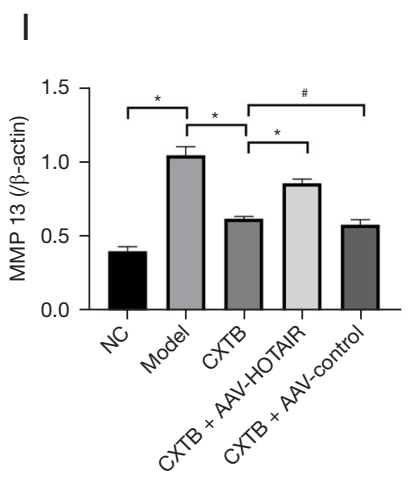

B

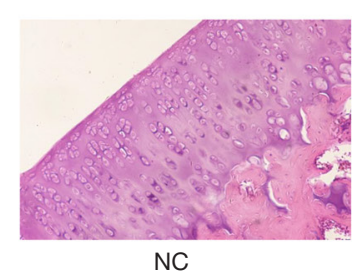

$\mathrm{F}$

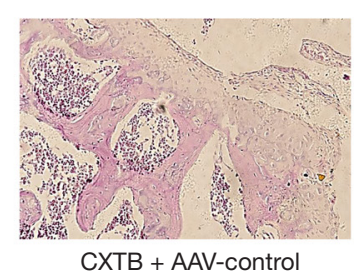

J

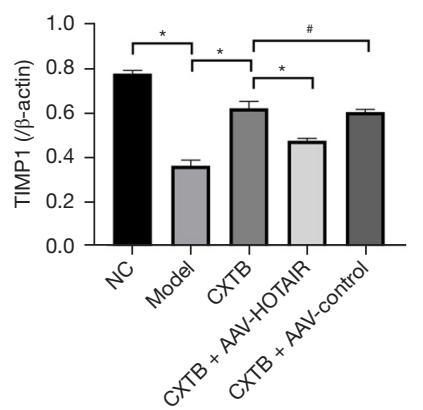

C

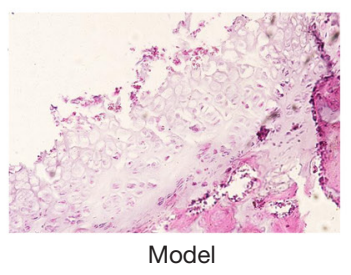

$\mathrm{D}$
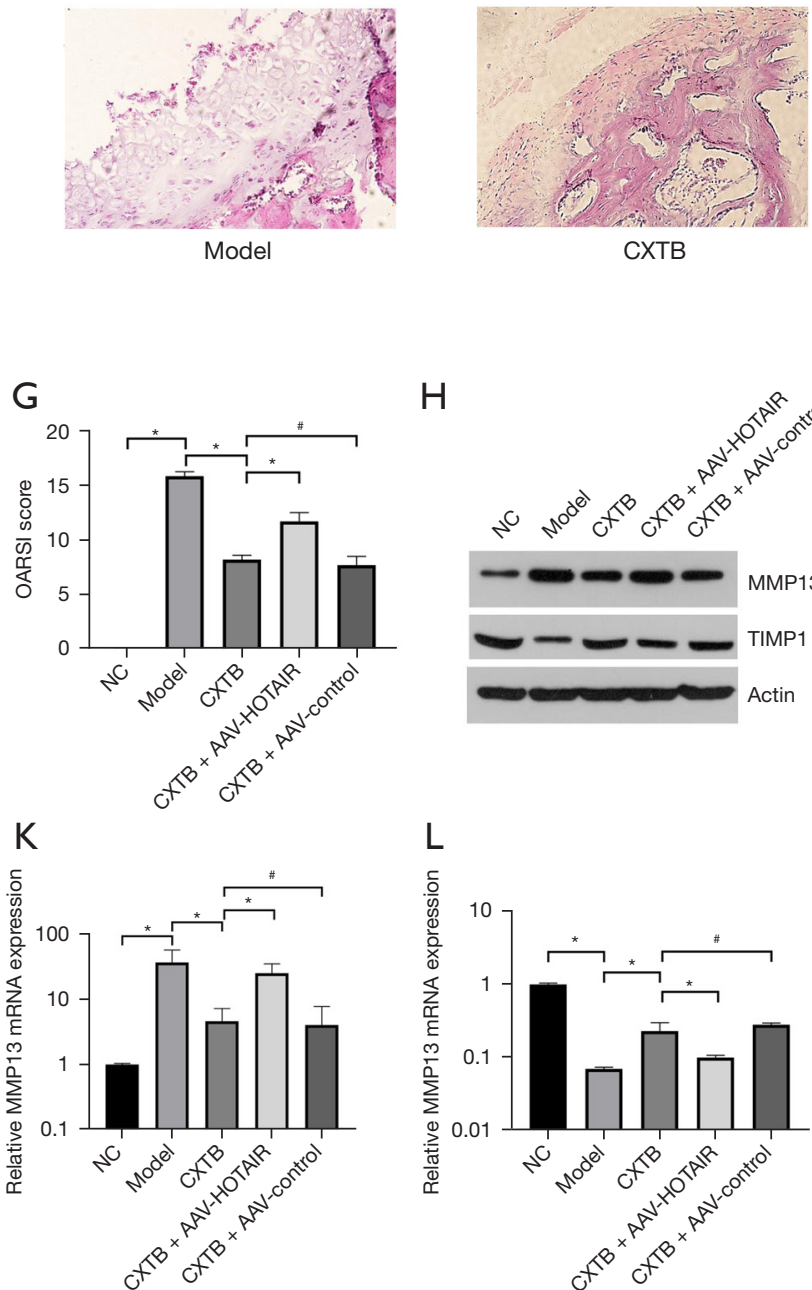

$\mathrm{H}$
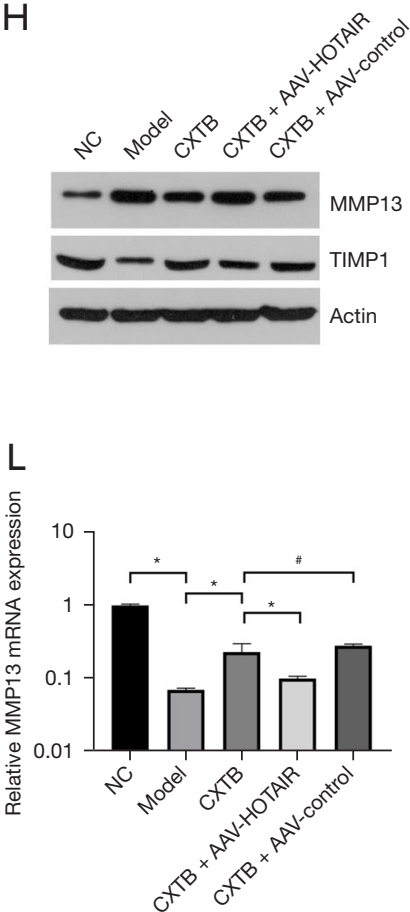

Figure 2 CXTB attenuates cartilage injury by downregulating the lncRNA HOTAIR. (A) The expression of lncRNA HOTAIR. (B-G) HE staining of the articular cartilage tissue $(\times 200)$ and the OARSI scores rats in different treatment groups. (H-L) The protein and mRNA expression of MMP13 and TIMP1. ${ }^{*} \mathrm{P}<0.05 ;{ }^{*} \mathrm{P}>0.05$. CXTB, cangxitongbi; lncRNA, long non-coding RNA; HOTAIR, Hox transcript antisense intergenic RNA; HE, hematoxylin and eosin; OARSI, Osteoarthritis Research Society International; MMP13, matrix metallopeptidase 13; TIMP1, tissue inhibitor matrix metalloproteinase 1; NC, negative control; AAV, adeno-associated virus.

chondrocytes of the NC rats had complete cell structure, abundant endoplasmic reticulum, and were concentrated in the perinuclear region. There were fewer autophagic vacuoles with double membrane structures in the model group compared with the NC group. Moreover, after treatment with CXTB, more autophagic vacuoles could be observed compared to the model group and the CXTB + AAV-HOTAIR group (Figure 4A-4E).

Western blot analysis (Figure $4 F, 4 G$ ) of the autophagyrelated proteins revealed that the expression of LC3-II was decreased in the articular cartilage tissues of the model rats compared to the $\mathrm{NC}$ rats $(\mathrm{P}<0.05)$. In the $\mathrm{CXTB}$ group, the expression of LC3-II was significantly elevated compared to the model group and the CXTB + AAV-HOTAIR group $(\mathrm{P}<0.05)$. Furthermore, there was no statistical difference in the LC3-II between the CXTB group and the CXTB + AAV control group $(\mathrm{P}>0.05)$. These results demonstrated that CXTB can enhance autophagy of KOA and may exert a protective role in the cartilage by downregulating lncRNA HOTAIR. 

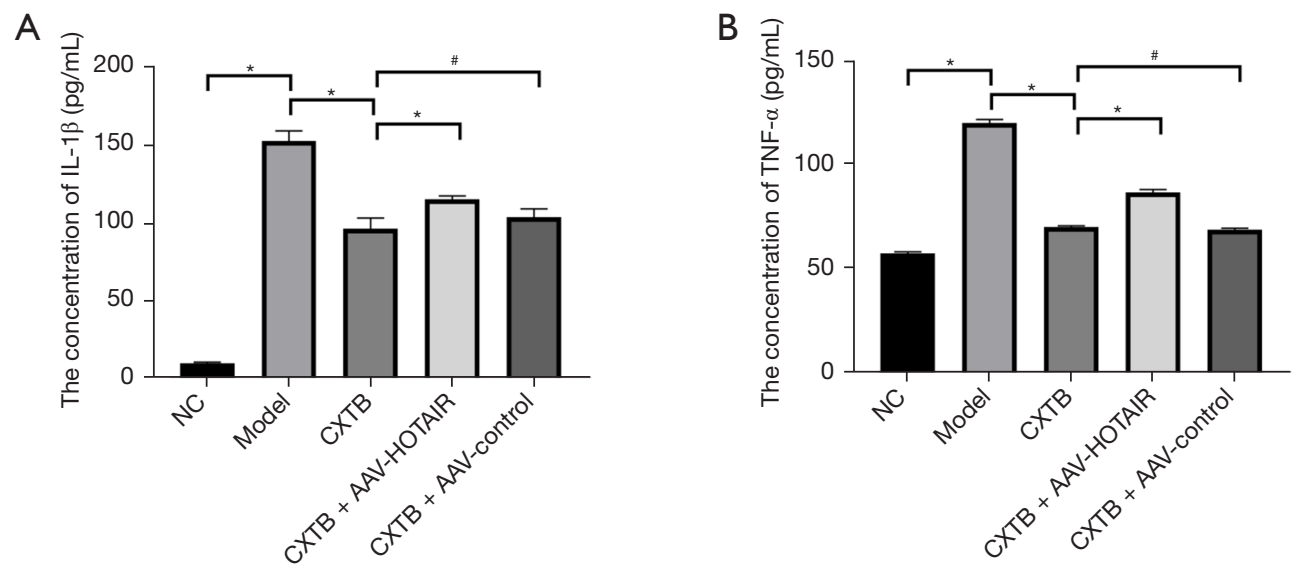

Figure 3 CXTB suppresses the inflammatory response by down-regulating lncRNA HOTAIR. (A,B) The expression levels of IL-1 $\beta$ and TNF- $\alpha$ in the serum of rats. ${ }^{*} \mathrm{P}<0.05$; ${ }^{*} \mathrm{P}>0.05$. CXTB, cangxitongbi; lncRNA, long non-coding RNA; HOTAIR, Hox transcript antisense intergenic RNA; IL, interleukin; TNF, tumor necrosis factor; NC, negative control; AAV, adeno-associated virus.

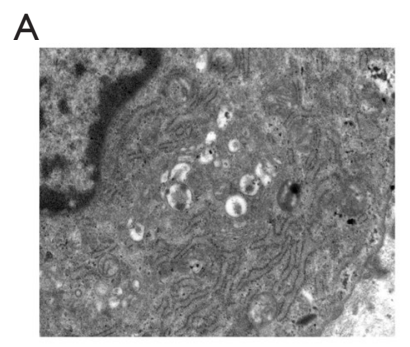

$\mathrm{NC}$

E

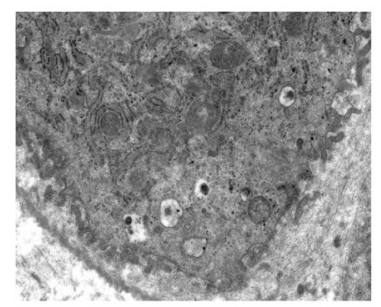

CXTB + AAV-control
B

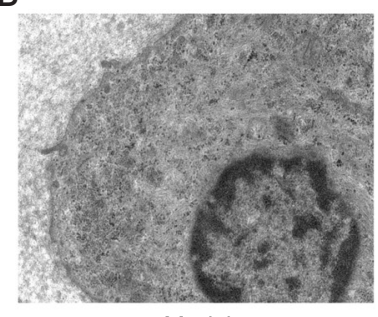

Model
C

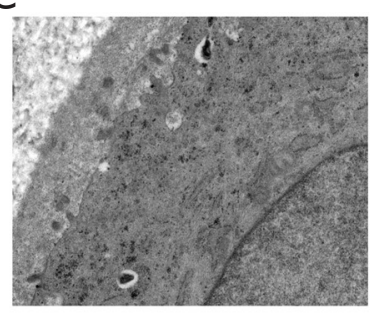

CXTB

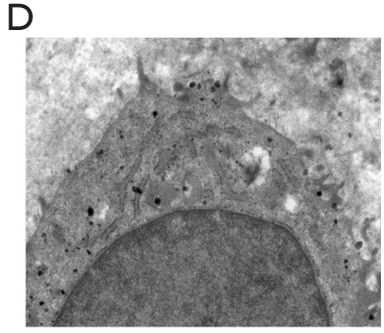

CXTB + AAV-HOTAIR
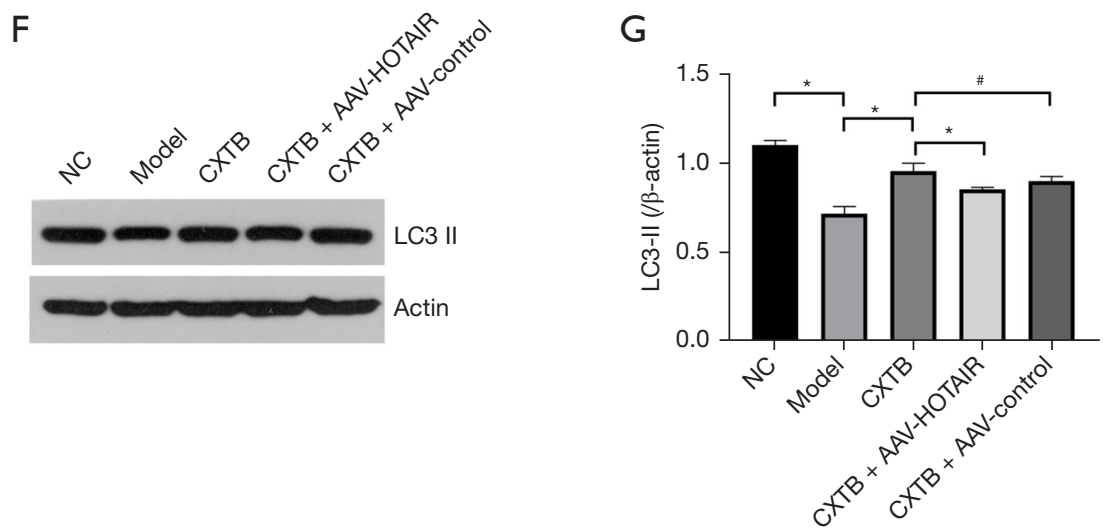

Figure 4 CXTB enhances autophagy by down-regulating lncRNA HOTAIR. (A-E) TEM images of the cartilage tissue $(\times 5,000)$. (F,G) The protein expression of LC3-II. * $\mathrm{P}<0.05$; ${ }^{*} \mathrm{P}>0.05$. CXTB, cangxitongbi; lncRNA, long non-coding RNA; HOTAIR, Hox transcript antisense intergenic RNA; TEM, transmission electron microscope; LC3, microtubule-associated protein light chain 3; NC, negative control; AAV, adeno-associated virus. 
A

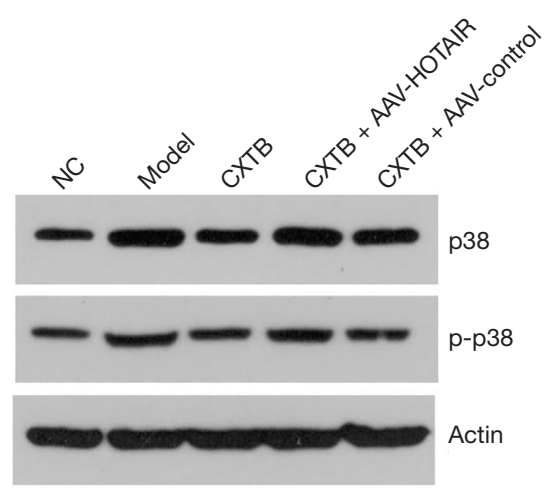

C

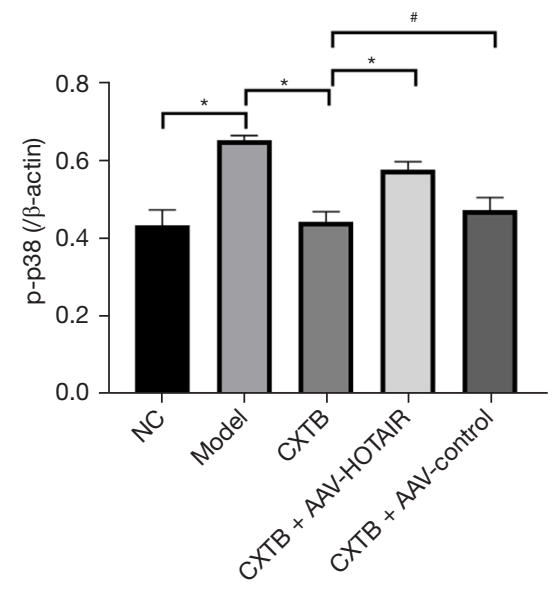

B
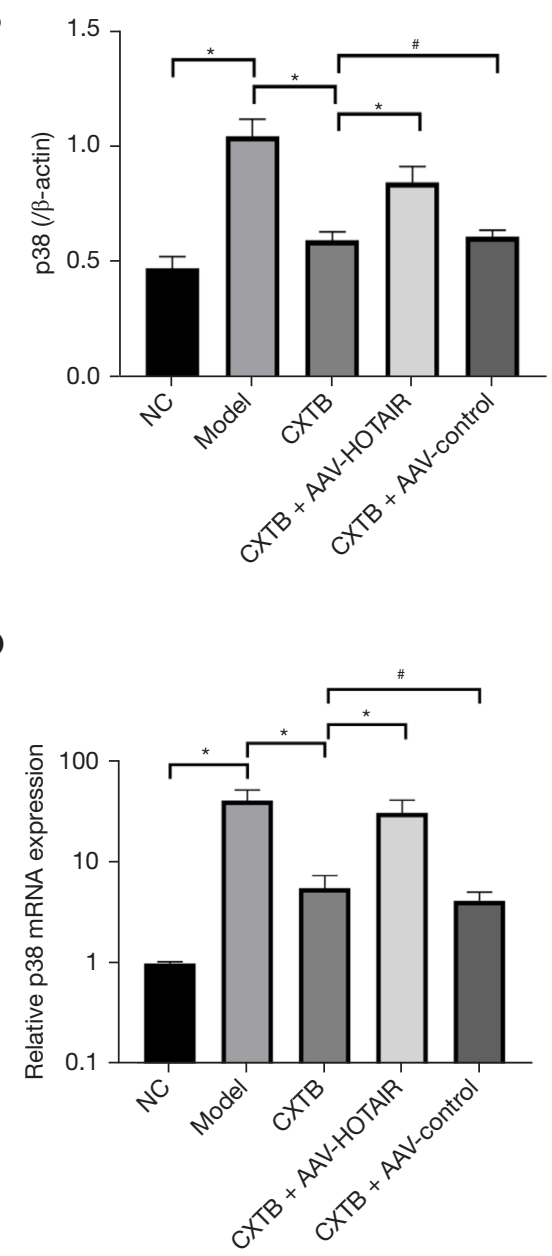

Figure 5 CXTB regulates the lncRNA HOTAIR/p38MAPK axis. (A-C) The protein expression of $\mathrm{p} 38$ and p-p38. (D) The mRNA expression of $\mathrm{p} 38$. ${ }^{*} \mathrm{P}<0.05 ;{ }^{*} \mathrm{P}>0.05$. CXTB, cangxitongbi; lncRNA, long non-coding RNA; HOTAIR, Hox transcript antisense intergenic RNA; p-p38, phosphorylated p38; NC, negative control; AAV, adeno-associated virus.

\section{CXTB regulates the IncRNA HOTAIR/p38MAPK axis}

To further investigate the mechanisms of CXTB, the interaction between IncRNA HOTAIR and the p38MAPK pathway was explored (Figure 5). The expression of p38 and p-p38 in the NC group and the CXTB group was significantly decreased compared to the model group $(\mathrm{P}<0.05)$. The expression of $\mathrm{p} 38$ and $\mathrm{p}-\mathrm{p} 38$ in the CXTB + AAV-HOTAIR group was higher than that of the CXTB group $(\mathrm{P}<0.05)$, while there was no significant difference between the CXTB group and the CXTB + AAV-control group $(\mathrm{P}>0.05)$ (Figure 5A-5C). To corroborate the above conclusions, qPCR was performed to measure the mRNA expression of $\mathrm{p} 38$ and the results were consistent with the Western blot analyses (Figure 5D). These results suggested that CXTB can regulate the lncRNA HOTAIR/p38MAPK axis, and the protective effects of CXTB on articular cartilage may be mediated through the lncRNA HOTAIR/ p38MAPK axis.

\section{Discussion}

CXTB is a patented Chinese drug widely used for the clinical treatment of KOA. However, the mechanisms of KOA remain unclear. This present study investigated the therapeutic effects of CXTB on KOA rats and demonstrated that CXTB can improve the structure of the injured cartilage and reduce the OARSI score. Previous studies have confirmed that MMP13 can degrade collagen, which 
is considered to be closely related to the pathogenesis of KOA (6). TIMP1 can specifically inhibit the function of MMP13 by inserting its inhibitory loop into the active site of the MMP13 catalytic domain (7). Therefore, MMP13 and TIMP1 are considered effective markers for evaluating the degree of cartilage damage $(8,9)$. Our results demonstrated that CXTB reduced the expression of MMP13 while elevating the expression of TIMP1, and this are responsible for the improved structure of the damaged cartilage. Taken together, the improvements in the damaged cartilage by CXTB is considered to be an important pharmacological effect.

IL- $1 \beta$ and TNF- $\alpha$ are common proinflammatory factors and main catabolic markers of KOA. Both have been shown to suppress the synthesis of the extra-cellular matrix (ECM) and increase apoptosis, thereby inhibiting the cartilage repair process (10). Furthermore, the ability of IL- $1 \beta$ and TNF- $\alpha$ to degrade cartilage is mediated by the elevated expression of MMP13 and the decreased expression of TIMP1 $(11,12)$. Therefore, the effects of CXTB on IL- $1 \beta$ and TNF- $\alpha$ were examined to corroborate the previous findings regarding MMP13 and TIMP1, and to investigate the anti-inflammatory role of CXTB. The results demonstrated that CXTB decreased the expression of IL- $1 \beta$ and TNF- $\alpha$, suggesting that CXTB can suppress the inflammatory response and further confirmed the observations related to MMP13 and TIMP1.

Autophagy, also known as type II programmed cell death and non-apoptotic cell death program (13), plays an important role in the progression of KOA (14). It is an intracellular reaction that can transform senescent and damaged organelles and proteins into substances needed by the cells and thus, autophagy is thought to exert a cartilage protective effect in KOA (15). Previous studies have shown that autophagy is decreased in KOA, accompanied by changes in the mitochondria and apoptosis (16). More importantly, older age, which is one of the triggers of KOA, can also lead to a decline in autophagy (14). During autophagy, part of the cytoplasm and organelles are sequestered in autophagosomes waiting to be degraded by lysosomes (17). Therefore, autophagy was examined by TEM and the autophagy-related proteins were evaluated by Western blot. The results demonstrated that the increased numbers of autophagosomes was accompanied by a higher LC3-II expression in the CXTB group, suggesting that CXTB may enhance the level of autophagy in KOA to protect damaged articular cartilage.

Recently, much research has focused on lncRNAs.
The lncRNA HOTAIR has been well-studied in tumor associated diseases (18), but there is a paucity of data regarding HOTAIR in KOA. Related studies have shown that HOTAIR is upregulated in KOA and closely associated with cartilage degeneration $(3,19)$. Moreover, upregulation of HOTAIR also elevated the levels of cartilage degenerative enzymes such as ADAMTS-5 and MMP13, as well as increasing chondrocyte apoptosis (20). Our results showed that CXTB can downregulate the expression of HOTAIR. To confirm the protective effect of CXTB on articular cartilage, the AAV-HOTAIR was constructed to overexpress HOTAIR in vivo. Ultimately, the results demonstrated a therapeutic effect of CXTB against KOA, including reduced cartilage damage, anti-inflammatory activity, and enhanced autophagy, all of which were reversed by overexpressing HOTAIR. This demonstrated the therapeutic effect of CXTB on KOA by targeting the downregulation of HOTAIR.

To further investigate the mechanism of HOTAIR in KOA, the downstream regulatory pathways of HOTAIR were examined. The p38MAPK pathway is an important pathway in $\mathrm{KOA}$ and is involved in inflammation and cartilage degradation (21-23). Furthermore, IL-1 $\beta$ and TNF- $\alpha$ can activate the p38MAPK pathway, leading to increased expression of MMP13 and ultimately, degradation of the cartilage ECM (24). Indeed, our results showed that CXTB can inhibit the p38MAPK pathway, and this inhibitory effect can be reversed by overexpression of HOTAIR.

\section{Conclusions}

This report demonstrated that CXTB can protect articular cartilage by suppressing inflammation, inhibiting cartilage ECM degradation, and enhancing autophagy via regulating the lncRNA HOTAIR/p38MAPK axis. CXTB may be a novel therapeutic drug for the treatment of patients with KOA.

\section{Acknowledgments}

Funding: This work was supported by the Natural Science Foundation of Shandong Province (ZR2020QH312, ZR2020MH099); and the Youth Program of the National Natural Science Foundation of China (82104897).

\section{Footnote}

Reporting Checklist: The authors have completed the 
ARRIVE reporting checklist. Available at https://dx.doi. org/10.21037/atm-21-6539

Data Sharing Statement: Available at https://dx.doi. org/10.21037/atm-21-6539

Conflicts of Interest: All authors have completed the ICMJE uniform disclosure form (available at https://dx.doi. org/10.21037/atm-21-6539). The authors have no conflicts of interest to declare.

Ethical Statement: The authors are accountable for all aspects of the work in ensuring that questions related to the accuracy or integrity of any part of the work are appropriately investigated and resolved. Animal experiments were approved by the Animal Ethics Committee of Affiliated Hospital of Shandong University of Traditional Chinese Medicine (AWE-2019-043), in compliance with Guide for the Care and Use of Laboratory Animals, 8th edition, for the care and use of animals.

Open Access Statement: This is an Open Access article distributed in accordance with the Creative Commons Attribution-NonCommercial-NoDerivs 4.0 International License (CC BY-NC-ND 4.0), which permits the noncommercial replication and distribution of the article with the strict proviso that no changes or edits are made and the original work is properly cited (including links to both the formal publication through the relevant DOI and the license). See: https://creativecommons.org/licenses/by-nc-nd/4.0/.

\section{References}

1. He CP, Jiang XC, Chen C, et al. The function of lncRNAs in the pathogenesis of osteoarthritis. Bone Joint Res 2021;10:122-33.

2. Wallace IJ, Worthington S, Felson DT, et al. Knee osteoarthritis has doubled in prevalence since the mid20th century. Proc Natl Acad Sci U S A 2017;114:9332-6.

3. He B, Jiang D. HOTAIR-induced apoptosis is mediated by sponging miR-130a-3p to repress chondrocyte autophagy in knee osteoarthritis. Cell Biol Int 2020;44:524-35.

4. Jiang YH, Zhang P, Tao Y, et al. Banxia Baizhu Tianma decoction attenuates obesity-related hypertension. J Ethnopharmacol 2021;266:113453.

5. Song XY, Xie WP, Zhang P, et al. Cangxitongbi capsule protects articular cartilage of the knee in rats by regulating ADAMTS-5. Ann Transl Med 2020;8:1511.
6. Yamamoto K, Okano H, Miyagawa W, et al. MMP-13 is constitutively produced in human chondrocytes and co-endocytosed with ADAMTS- 5 and TIMP- 3 by the endocytic receptor LRP1. Matrix Biol 2016;56:57-73.

7. Li Y, Tang L, Duan Y, et al. Upregulation of MMP13 and TIMP-1 expression in response to mechanical strain in MC3T3-E1 osteoblastic cells. BMC Res Notes 2010;3:309.

8. Zhang W, Zhang C, Luo C, et al. Design, cyclization, and optimization of MMP13-TIMP1 interaction-derived selfinhibitory peptides against chondrocyte senescence in osteoarthritis. Int J Biol Macromol 2019;121:921-9.

9. Salerno A, Brady K, Rikkers M, et al. MMP13 and TIMP1 are functional markers for two different potential modes of action by mesenchymal stem/stromal cells when treating osteoarthritis. Stem Cells 2020;38:1438-53.

10. Pujol JP, Chadjichristos C, Legendre F, et al. Interleukin-1 and transforming growth factor-beta 1 as crucial factors in osteoarthritic cartilage metabolism. Connect Tissue Res 2008;49:293-7.

11. Pathak NN, Lingaraju MC, Balaganur V, et al. Antiinflammatory and chondroprotective effects of atorvastatin in a cartilage explant model of osteoarthritis. Inflamm Res 2015;64:161-9.

12. Bokhari RA, Tantowi NACA, Lau SF, et al. Java Tea (Orthosiphon stamineus) protected against osteoarthritis by mitigating inflammation and cartilage degradation: a preclinical study. Inflammopharmacology 2018;26:939-49.

13. Musumeci G, Szychlinska MA, Mobasheri A. Age-related degeneration of articular cartilage in the pathogenesis of osteoarthritis: molecular markers of senescent chondrocytes. Histol Histopathol 2015;30:1-12.

14. Luo P, Gao F, Niu D, et al. The Role of Autophagy in Chondrocyte Metabolism and Osteoarthritis: A Comprehensive Research Review. Biomed Res Int 2019;2019:5171602.

15. Chang J, Wang W, Zhang H, et al. The dual role of autophagy in chondrocyte responses in the pathogenesis of articular cartilage degeneration in osteoarthritis. Int J Mol Med 2013;32:1311-8.

16. Wu C, Zheng J, Yao X, et al. Defective autophagy in chondrocytes with Kashin-Beck disease but higher than osteoarthritis. Osteoarthritis Cartilage 2014;22:1936-46.

17. Musumeci G, Castrogiovanni P, Trovato FM, et al. Biomarkers of Chondrocyte Apoptosis and Autophagy in Osteoarthritis. Int J Mol Sci 2015;16:20560-75.

18. Chen H, Qi J, Bi Q, et al. Expression profile of long noncoding RNA (HOTAIR) and its predicted target miR- 


\section{Page 10 of 10}

$17-3 \mathrm{p}$ in LPS-induced inflammatory injury in human articular chondrocyte C28/I2 cells. Int J Clin Exp Pathol 2017;10:9146-57.

19. Hu J, Wang Z, Shan Y, et al. Long non-coding RNA HOTAIR promotes osteoarthritis progression via miR-175 p/FUT2/ $\beta$-catenin axis. Cell Death Dis 2018;9:711.

20. Zhang C, Wang P, Jiang P, et al. Upregulation of lncRNA HOTAIR contributes to IL-1 $\beta$-induced MMP overexpression and chondrocytes apoptosis in temporomandibular joint osteoarthritis. Gene 2016;586:248-53.

21. Lei J, Fu Y, Zhuang Y, et al. LncRNA SNHG1 alleviates IL-1 $\beta$-induced osteoarthritis by inhibiting miR-16-5pmediated p38 MAPK and NF- $\mathrm{NB}$ signaling pathways. Biosci Rep 2019;39:BSR20191523.

Cite this article as: Song XY, Zhao M, Zhang $\mathrm{P}$, Yang LS, Bi RX, Xie WP. Cangxitongbi capsules protect the articular cartilage in the rat knee through the long noncoding RNA HOTAIR/p38MAPK pathway. Ann Transl Med 2022;10(1):23. doi: 10.21037/atm-21-6539

\section{Song et al. CXTB regulates IncRNA HOTAIR/p38MAPK in vivo}

22. Wang D, Qiao J, Zhao X, et al. Thymoquinone Inhibits IL-1 $\beta$-Induced Inflammation in Human Osteoarthritis Chondrocytes by Suppressing NF- $\mathrm{B}$ and MAPKs Signaling Pathway. Inflammation 2015;38:2235-41.

23. Liao CR, Wang SN, Zhu SY, et al. Advanced oxidation protein products increase TNF- $\alpha$ and IL- $1 \beta$ expression in chondrocytes via NADPH oxidase 4 and accelerate cartilage degeneration in osteoarthritis progression. Redox Biol 2020;28:101306.

24. Xue J, Ye J, Xia Z, et al. Effect of luteolin on apoptosis, MAPK and JNK signaling pathways in guinea pig chondrocyte with osteoarthritis. Cell Mol Biol (Noisy-legrand) 2019;65:91-5.

(English Language Editor: J. Teoh) 